\title{
Slow and Fast Light in Semiconductor Optical Amplifiers for Microwave Photonics Applications
}

\author{
Perrine Berger ${ }^{1}$, Jérôme Bourderionnet ${ }^{2}$, Daniel Dolfi ${ }^{3}$, \\ Fabien Bretenaker ${ }^{4}$ and Mehdi Alouini ${ }^{5}$ \\ 1,2,3 Thales Research and Technology \\ ${ }^{4}$ Laboratoire Aimé Cotton, CNRS - Université Paris Sud \\ ${ }^{5}$ Institut de Physique de Rennes
}

France

\section{Introduction}

The generation of continuously tunable optical delays and tunable phase shifts is a key element in microwave photonics. Among the targeted applications, one can quote the filtering of microwave signals, the synchronization of optoelectronics oscillators, and the control of optically fed phased array antennas. With these applications in view, large efforts are currently done in order to develop delay lines and phase shifters based on slow and fast light effects. To date, one of the most mature approaches for integration in real field systems is that based on Coherent Population Oscillations (CPO) in Semiconductor Optical Amplifiers (SOAs). This approach offers compactness, continuous tunability of the delay or phase shift through injected current control, and possible high-level parallelism.

Slow and fast light capability of semiconductor devices has been first studied in the past decade while bearing in mind the delays, the phase shifts and the bandwidth they can offer. Consequently, in a first section, we present the recent advances in architectures based on slow and fast light in SOAs for microwave photonics applications. After a brief introduction about microwave photonics, we present the physical interpretation of the different architectures proposed in the literature. We point out the underlying physics, common to these architectures, and evidence the advantages and drawbacks of each of them. However, within the scope of integration in a realistic radar system, it is also required to study the impact of these slow and fast light architectures on the performances of the microwave photonics link. In particular, the RF transfer function, the generation of spurious signals by harmonic and intermodulation products, and the intensity noise, have to be studied in order to compute the Spurious Free Dynamic Range (SFDR), a key characteristic in microwave photonics. Consequently, in a second section, we present the tools to simulate and understand the RF transfer function, the generation of spurious signals through harmonic distortion and intermodulation products, and the intensity noise at the output of a SOA.

In a third section, we use the models presented in the previous part in order to investigate the dynamic range of a microwave photonics link including an architecture based on slow and fast light in SOAs. We focus on the architecture using a SOA followed by an optical filter. The models are experimentally validated and the influence on the microwave photonics link is discussed. 


\section{Background and context}

\subsection{Slow and fast light for microwave photonics}

This section presents microwave photonics and explains why slow and fast light can be useful for this applied research field.

\subsubsection{Microwave photonics link including a slow and fast light device}

Microwave photonics realizes processing of microwave signals $(\Omega / 2 \pi \simeq 0.1-35 \mathrm{GHz})$ in the optical domain using photonic devices. Indeed optics offer some advantages compared to electronics for the addressing and processing of microwave signals: the most sticking asset is the low loss transport along an optical fiber $(0.2 \mathrm{~dB} / \mathrm{km})$ compared to a coaxial cable $(1000 \mathrm{~dB} / \mathrm{km}$ !). As illustrated in Fig. 1, the basic architecture of a microwave photonics link is composed of a laser, which creates the optical carrier $\left(\lambda_{0} \simeq 1.5 \mu \mathrm{m}\right)$. The optical carrier is modulated by the microwave signal either directly, or through an external Mach Zehnder modulator. Optical devices (represented on Fig. 1 by "slow and fast light device") process the modulated carrier. At the end of the link, a fast photodiode retrieves the processed microwave signal.

The aim of our study is to introduce a slow and fast light device in a microwave photonics link, as it is represented in Fig. 1. Let us consider a monochromatic microwave signal (whose angular frequency is $\Omega$ ) and a linear modulator, the optical field $E$ after the modulator is then composed of the optical carrier $E_{0}(z) e^{-i \omega_{0} t}$ and two sidebands $E_{1}(z) e^{-i\left(\omega_{0}+\Omega\right) t}+$ $E_{-1}(z) e^{i\left(\omega_{0}-\Omega\right) t}$.

After propagation through the slow and fast light device, the microwave signal retrieved by the photodiode, at the angular frequency $\Omega$, is:

$$
\begin{aligned}
M_{1}^{\text {OUT }} & =\sum_{\omega_{p}-\omega_{q}=\Omega} E_{p} E_{q}^{*}, \\
& =E_{1} E_{0}^{*} e^{i\left(k_{1}-k_{0}\right) L}+E_{-1}^{*} E_{0} e^{i\left(k_{0}-k_{-1}\right) L}, \text { when both sidebands are detected, } \\
& =E_{1} E_{0}^{*} e^{i\left(k_{1}-k_{0}\right) L} \text { or } E_{-1}^{*} E_{0} e^{i\left(k_{0}-k_{-1}\right) L}, \text { when only one sideband is detected, }
\end{aligned}
$$

where $L$ is the length of the dispersive medium (described by $k_{i}=k\left(\omega_{i}\right)$ ).

To characterize a microwave photonics link, we study the microwave transfer function through the complex parameter $S_{21}=\frac{M_{1}^{\text {OUT }}}{M_{1}^{I N}}$, whose magnitude and phase can be expressed
as:

$$
\begin{aligned}
\left|S_{21}\right| & =G^{2}, \\
\arg \left(S_{21}\right) & =\Delta k L,
\end{aligned}
$$

where $G$ is the optical gain of the link, and $\Delta k L$ the phase shift introduced by the dispersive medium (for example $\Delta k=k\left(\omega_{0}+\Omega\right)-k\left(\omega_{0}\right)$ when only the sideband $E_{1}$ is detected). Consequently, by governing the dispersion and in particular the optical group velocity $v_{g}=$ $\frac{d \omega}{d k}$ of the slow and fast light medium, it is possible to induce a controlled phase shift or delay on the retrieved microwave signal, which is an important function in microwave photonics, as it is illustrated in the following sections. 


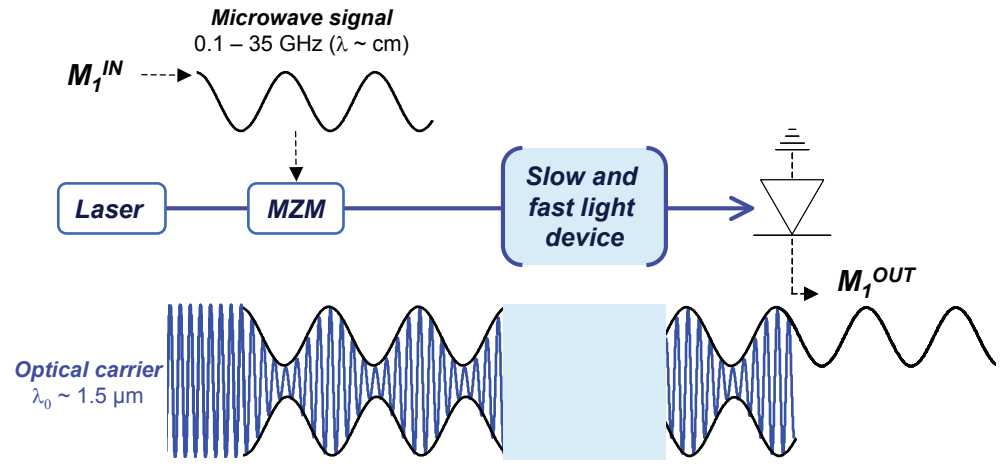

Fig. 1. Microwave photonics link including a slow and fast light device. MZM=Mach Zehnder Modulator.

\subsubsection{Tunable true time delay line for microwave photonics}

If a tunable true time delay is introduced on a microwave signal at the angular frequency $\Omega$, the phase shift introduced by the slow and fast light medium must be:

$$
\Delta k L=\tau \Omega \text { with } \tau \text { tunable. }
$$

This definition is illustrated on Fig. 2.

The generation of tunable true time delays over a large instantaneous RF bandwidth $\Delta f_{R F}$ is a key function in the processing of microwave signals, where the instantaneous bandwidth $\Delta f_{R F}$ will reach $10 \%$ to $30 \%$ of the operating frequency $f_{\text {operating. }}$ To illustrate this point, let us focus on the addressing of active antennas. Active antennas offer a better precision of the radiation direction, a better gain, and lower secondary lobes than classic antennas.They are composed of a matrix of radiating elements whose input signals can be controlled in magnitude and phase. The radiating pattern and the main radiating direction are controlled through the phase shifter of each radiating element. The phases applied to the different emitters define the phase plane in which all the RF signals constructively interfere. The main radiating direction is then perpendicular to this plan. Angling the beam is then equivalent to tuning the phase plane, which is controlled by the RF phase shifters. However, the delays introduced by these phase shifters are frequency dependent: consequently, a change in frequency introduces an uncontrolled beam squint. Tunable true time delay lines is an answer to these problems.

The generation of true time delays, tunable up to the nanosecond (or larger), on an instantaneous bandwidth reaching the GHz range is necessary. Optical solutions have already been proposed, by geometrically modifying the optical path. However these techniques does not match the real field requirements because of the weight, the cost and the limited number of achievable delays. Slow and fast light media could offer a complementary solution, by offering a fast, continuous, and precise control of delays.

\subsubsection{Tunable phase shifter for microwave photonics}

A microwave photonics including a slow and fast light medium can also be used as an optically tunable RF phase shifter. The phase shift introduced by the slow and fast light medium must be:

$$
\Delta k L=\phi \text { covering }[0,2 \pi]
$$




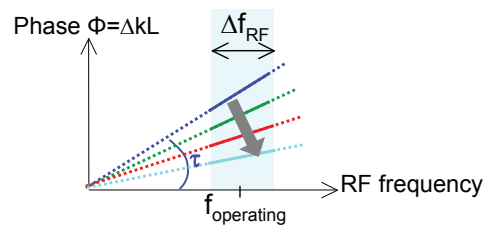

(a) Tunable true time delay

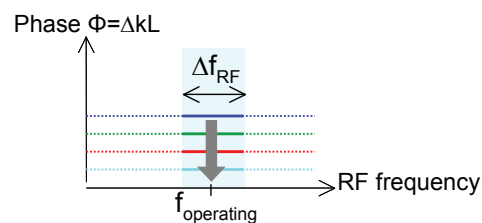

(b) RF Phase shifter

Fig. 2. (a) Illustration of tunable true time delay. (b) Illustration of RF phase shifter.

This definition is illustrated on Fig. 2.

This component exists in the microwave world (contrary to the true time delay lines). However, more and more RF functions are developed by photonic means. Consequently, tunable RF phase shifter in the optical domain is required in order to avoid useless Optical/Electronical and Electronical/Optical conversions. Among the targeted applications, one can quote the filtering of microwave signals: on each arm a phase shifter over an instantaneous bandwidth $\Delta f_{R F}$ is required; or optoelectronics oscillators, either for synchronization or tunability: in this case, only a phase shifter at the operating frequency is required.

To date, one of the most mature approaches of slow and fast light medium for integration in real field systems is that based on Coherent Population Oscillations (CPO) in semiconductor optical amplifiers (SOAs). This approach offers compactness, continuous tunability of the delay or phase shift through injected current control, and possible high-level parallelism. We present in the following paragraphs the basic concepts of this technique.

\subsection{Coherent Population Oscillations (CPO) in Semiconductor Optical Amplifier (SOA)}

This section introduces the basic concepts and the main equations involved in Coherent Population Oscillations (CPO) in a Semiconductor Optical Amplifier (SOA), and explains how this phenomenon induces slow and fast light. We describe the SOA behavior by a phenomenological model initially described for semiconductor lasers by Agrawal \& Dutta (1993), and which was initially used to describe CPO in SOA (Agrawal, 1988; Mørk et al., 2005). It gives a good insight in the involved phenomena. However, this model, initially developed for lasers, must be cautiously used in the case of SOAs: its limitations are discussed in section 4, where a more rigorous model is developed. From this phenomenological model, we derive the equations of propagation and analyze the dispersion properties and in particular the changes in group velocity induced by CPO.

\subsubsection{Phenomenological model of SOA}

This phenomenological model of SOA, well described by Agrawal \& Dutta (1993) is based on experimental observations of the behavior of semiconductor lasers.

The main assumption of the model consists in considering that the variations of the material gain $g$ and optical index $n$, caused by a small variation of the carrier density $\Delta N$, are proportional to $\Delta N$. The material gain $g$ and the optical index $n$ can thus be expressed as:

$$
\begin{aligned}
& g=\bar{g}+\Delta g, \\
& n=\bar{n}+\Delta n,
\end{aligned}
$$


with $\bar{N}$ and $\bar{g}=g(\bar{N})$ the static carrier density and gain, $\bar{n}=n(\overline{(N)})$, the static optical index, and $\Delta g$ and $\Delta n$ the variations of the material gain and the optical index caused by a small change $\Delta N$ of the carrier density. We assume then:

$$
\Delta g \propto \Delta N \text { and } \Delta n \propto \Delta N
$$

We introduce the differential gain $a$ :

$$
\Delta g=a \Delta N
$$

and the linewidth enhancement factor $\alpha$ introduced by Henry (1982) to model the index gain coupling in semiconductor material: $\alpha=-2 k_{0} \frac{\Delta n}{\Gamma \Delta g}$. Then the variation $\Delta n$ can be written as:

$$
\Delta n=-\frac{\alpha}{2 k_{0}} \Gamma \Delta g
$$

with $k_{0}=\frac{\omega}{c}$, and $\Gamma$ the confinement factor. We complete this phenomenological model with a rate equation, which incorporates all the mechanisms by which the carriers are generated or lost in the active region:

$$
\frac{d N}{d t}=\frac{I}{q V}-\frac{N}{\tau_{s}}-\frac{g|E|^{2}}{\hbar \omega_{0}},
$$

where $I$ is the injected current, $|E|^{2}$ is the optical intensity inside the SOA, $\tau_{s}$ is the carrier lifetime, $V$ is the volume of the active region, $q$ is the elementary charge, and $\omega_{0}$ is the angular frequency of the optical carrier $E_{0}$.

\subsubsection{Coherent population oscillations}

CPOs are induced by an optical carrier which is modulated in intensity at the angular frequency $\Omega:|E|^{2}=M_{0}+M_{1} e^{-i \Omega t}+$ c.c.. Note that the injected current can also be modulated (see section 3.3).

If the optical carrier power is large enough, it implies a gain saturation. Indeed, the rate equation (11) shows that oscillations of the carriers (CPO) are induced : $N=\bar{N}+\Delta N e^{-i \Omega t}+$ c.c.. At the first order, we can assume a linear variation of the gain and optical index (Eq. 8):

$$
\begin{aligned}
& g=\bar{g}+\Delta g e^{-i \Omega t}+\text { c.c. } \\
& n=\bar{n}+\Delta n e^{-i \Omega t}+\text { c.c. }
\end{aligned}
$$

Finally the Eqs. 9 and 11 lead to the expression of the gain variation $\Delta g$ (and then $\Delta n$ thanks to Eq. 10):

$$
\Delta g=\frac{A}{1+M_{0} / U_{s}-i \Omega \tau_{s}},
$$

where $M_{0}$ is the DC component of the optical intensity, $U_{s}$ the saturation intensity, and for sake of clarity, $A$ is considered here as a constant (for example $A=-\bar{g} M_{1} / U_{s}$ when only the optical intensity is modulated, see section 4 ).

The optical index is then time and frequency dependent due to the CPO, which implies slow and fast propagation of the light, as we explain in the following paragraphs. 


\subsubsection{Equations of propagation}

The optical field $\mathcal{E}(z, t)$ verifies the following wave equation:

$$
\frac{\partial^{2}}{\partial^{2} z} \mathcal{E}(z, t)=\frac{1}{c^{2}} \frac{\partial^{2}}{\partial^{2} t} \epsilon_{s \mathcal{C}} \mathcal{E}(z, t)
$$

where $\epsilon_{S C}$ is the relative permittivity.

We expand the optical field as $\mathcal{E}(z, t)=\Re\left\{\sum_{p} E_{p}(z) e^{i\left(\tilde{\beta}_{p} z-\omega_{p} t\right)}\right\}$, where $\tilde{\beta}_{p}$ is the complex propagation constant. $E_{0}$ accounts for the optical carrier, and $E_{ \pm 1}$ the modulation sidebands at $\omega_{ \pm 1}=\omega_{0} \pm \Omega$. The complex propagation constant can be expressed as:

$$
\tilde{\beta}=k_{0} \tilde{\mu}=k_{0} \sqrt{\epsilon_{s c}},
$$

with $k_{0}=\frac{\omega}{c}$ and $\tilde{\mu}$ the complex optical index, which can be written as:

$$
\tilde{\mu}=n+i \frac{-\Gamma g+\gamma}{2 k_{0}},
$$

where $n$ is the real refractive optical index, and $\gamma$ holds for internal losses. From Eqs. 12, 13 and 17 , the complex optical index $\tilde{\mu}$ can be expanded as: $\tilde{\mu}=\tilde{\mu}_{0}+\Delta \tilde{\mu} e^{-i \Omega t}+$ c.c., with:

$$
\begin{aligned}
\tilde{\mu}_{0} & =\bar{n}+\frac{i}{2 k_{0}}(-\Gamma \bar{g}+\gamma), \\
\Delta \tilde{\mu} & =-\frac{\alpha+i}{2 k_{0}} \Gamma \Delta g .
\end{aligned}
$$

Lastly, we derive the equations of propagation from Eqs. 15, 18 and $\epsilon_{S C}=\tilde{\mu}^{2}$ :

$$
\begin{aligned}
\frac{d E_{0}}{d z} & =\frac{1}{2}(\Gamma \bar{g}-\gamma) E_{0}, \\
\frac{d E_{1}}{d z} & =\frac{1}{2}(\Gamma \bar{g}-\gamma) E_{1}+\frac{1-i \alpha}{2} \Gamma \Delta g E_{0}, \\
\frac{d E_{-1}}{d z} & =\frac{1}{2}(\Gamma \bar{g}-\gamma) E_{-1}+\frac{1-i \alpha}{2} \Gamma \Delta g^{*} E_{0} .
\end{aligned}
$$

\subsubsection{Slow and fast light induced by CPO}

We have shown in the previous paragraph that the modulation of the optical intensity leads to $\mathrm{CPO}$, which induces a frequency dependence of the complex optical index. We illustrate here how it induces slow and fast light.

We define the real, $n_{r}$, and imaginary, $n_{i m}$, parts of the index: $\tilde{\mu}=n_{r}+i n_{i m}$. The Eqs. 14 and 18 lead to the following expressions of the variations of the real and imaginary parts of the optical index, induced by CPO:

$$
\begin{gathered}
\Delta n_{r}=\Re\{\Delta \tilde{\mu}\}=-\frac{c A}{2 w} \frac{\alpha\left(1+M_{0} / U_{s}\right)-\Omega \tau_{s}}{\left(1+M_{0} / U_{s}\right)^{2}+\left(\Omega \tau_{s}\right)^{2}}, \\
\Delta n_{i m}=\Im\{\Delta \tilde{\mu}\}=-\frac{c A}{2 w} \frac{\left(1+M_{0} / U_{s}\right)+\alpha \Omega \tau_{s}}{\left(1+M_{0} / U_{s}\right)^{2}+\left(\Omega \tau_{s}\right)^{2}},
\end{gathered}
$$

where $\Omega$ is the angular frequency of the $\mathrm{CPO}, w$ is the angular frequency of the considered optical field component, and $w=w_{0}+\Omega$ with $w_{0}$ the angular frequency of the optical carrier. 

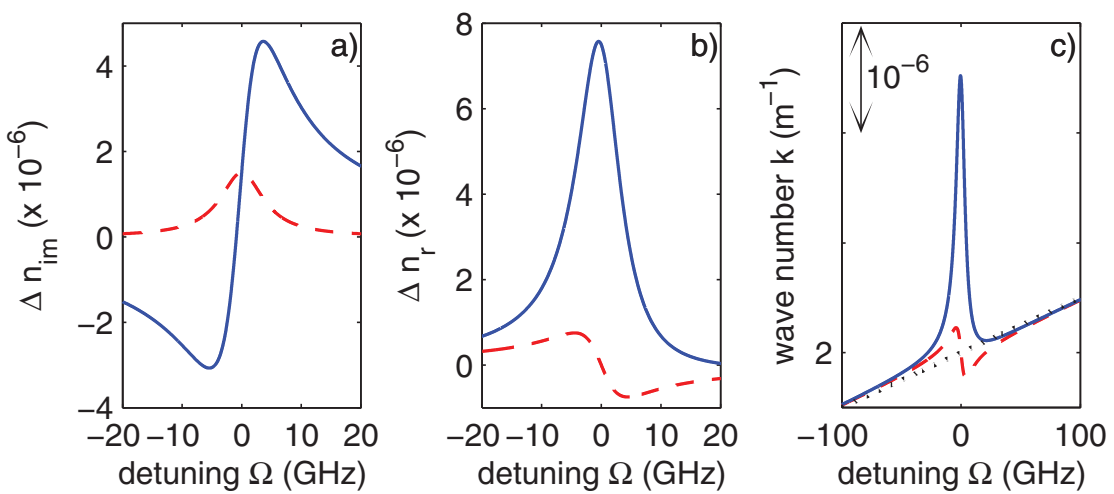

Fig. 3. Variations of (a) the imaginary part $\Delta n_{i m}$ of the complex optical index (proportional to the absorption), (b) the real part $\Delta n_{r}$ of the complex optical index, and (c) the wave number $k$. In blue, for semiconductor material (where $\alpha \neq 0$ ), and in dashed red line, for an equivalent 2-level atomic resonance (where $\alpha=0$ ). Parameters: $\alpha=5$ (blue line) or $\alpha=0$ (red dashed line), $M_{0} / U_{s}=1, A=-4000 m^{-1}, \tau_{s}=450 p s, n_{s c}=3, \lambda_{0}=1.5 \mu m$.

The variations of the imaginary $\Delta n_{i m}$ and real $\Delta n_{r}$ parts of the optical index are displayed on Fig.3(a) and (b). $\Delta n_{i m}$ and $\Delta n_{r}$ are related by the Kramers-Kronig relationship. We illustrate that for semiconductor material, due to the coupling index-gain (modeled by the factor $\alpha$ ), the variation of the imaginary $\Delta n_{i m}$ optical index, proportional to the variation of the absorption, is asymmetric: it has been first observed by Bogatov et al. (1975). The variation of the optical index with respect to the frequency is then very different from the case of 2-level atomic resonance (represented in dashed red line in Fig. 3).

The frequency dependence of the optical index leads to a strong dispersion in the vicinity of the frequency of the optical carrier: as illustrated on Fig.3(c), the real wave number $k(\omega)=$ $\frac{n(\omega) \omega}{c}$ differs then from the "normal" refraction $\frac{n_{s c} \omega}{c}$ (represented by the black dotted straight line). This is associated with a variation of the group velocity of the light, which can be defined as $v_{g}=\frac{d \omega}{d k}$. Consequently, $\mathrm{CPO}$ create here $(A<0)$ "fast light" for positive detuning $\Omega>0$ $\left(\frac{d k}{d \omega}<0\right)$, and "slow light" for negative detuning $\Omega<0\left(\frac{d k}{d \omega}>0\right)$. In comparison, a 2-level atomic resonance create essentially fast light at low detuning: $|\Omega|<$ a few GHz. As the amplitude of CPO is controlled by either the input optical power $\left(M_{0}\right)$ or the injected current (though the parameter $A$ ), the group velocity can be tuned and controlled, which opens the possibility to conceive optical delay lines and optical phase shifters for microwave signals, as we will explain it in the following part.

The arrival time $\tau$ (to a detector for example) of a signal propagating through this slow and fast light medium has to be carefully handled. Indeed it is equal to the group delay simply defined as $\tau_{g}=\left.L \frac{d k}{d \omega}\right|_{\bar{\omega}}$, with $\bar{\omega}$ the central optical frequency of the signal, only if the modulation before the photodiode is Single-SideBand (SSB), and if the bandwidth of the signal is smaller than the $\mathrm{CPO}$ bandwidth $(<1 \mathrm{GHz})$. We will highlight this last point in the following part. For further details on the definition of the arrival time for optical pulses, you can refer to (Peatross et al., 2000). 


\section{Advances in architectures based on slow and fast light in SOAs for microwave photonics applications}

In the previous part, we saw the underlying physics of the slow and fast light generated by Coherent Population Oscillations (CPO) in SOAs. We present here the different architectures based on this phenomenon, which are proposed for microwave photonics applications. In the first section, we present an architecture of an optical delay line. In the second section, we show that by changing the modulation format before the photodiode, the latter architecture becomes an optical phase shifter for microwave signals. Lastly, we present an alternative set-up to realize an optical RF phase shifter, by using forced CPO.

\subsection{SOA-based optical delay line}

\subsubsection{Set-up, experimental results and equations}

This architecture is the first proposed in literature (Mørk et al., 2005; Pesala et al., 2006). The set-up is quite simple: the slow and fast light device (represented in Fig. 1) included in the microwave link is a single SOA. The corresponding experimental results are presented on Fig. 4. The microwave gain $\left|S_{21}\right|$ present a high pass filter behavior, usually observed in SOAs (Boula-Picard et al., 2005), and is associated to an interesting phase shift, which presents a linear variation at low frequency, with a slope tunable through the injected current or the optical input power: a key characteristic to set up a tunable delay line (see Fig. 2).

These results are not in adequation with the expected behavior of a semiconductor material in which the coupling index-gain is significant $(\alpha \neq 0)$ (displayed in Fig. 3), but it is similar to the material where $\alpha=0$ : indeed a negative delay (which is then an advance, associated to a so called "fast light") is detected at low frequency $(<\mathrm{GHz})$. However it can be easily explained by the double-sideband modulation format before the detector. Indeed, as both the modulation sidebands are detected, the RF retrieved signal at the angular frequency $\Omega$ is $M_{1}=E_{1} E_{0}^{*}+E_{-1} E_{0}^{*}$, and Eq. 19 leads to:

$$
\frac{d M_{1}}{d z}=[-\gamma+\Gamma \bar{g}] M_{1}+\Gamma \Delta g M_{0}
$$

with $\Delta g$ deduced from the rate equation (11):

$$
\Delta g=-\frac{\bar{g} M_{1} / U_{s}}{1+M_{0} / U_{s}-i \Omega \tau_{s}}
$$

with $U_{s}=\frac{\hbar \omega}{\Gamma a \tau_{s}}$. We notice then that the contribution of the coupling gain-index is canceled out when both the sidebands are detected, which explains that the gain and phase shift are similar to a material where $\alpha=0$.

\subsubsection{Analysis of the different contributions. Physical interpretation}

We integrate Eq. 22 over a small slice $d z$, whose length is noted $L$, to derive an analytical expression of the RF transfer function $S_{21}=\frac{M_{1}(L)}{M_{1}(0)}$ :

$$
\frac{M_{1}(L)}{M_{1}(0)}=\mathcal{A}+\frac{1}{2} \mathcal{G}^{o p t}
$$

with $\mathcal{A}=1-\gamma L+\Gamma \bar{g} L$ corresponding to the optical amplification (average RF gain) and $\mathcal{G}^{\text {opt }}$ the contribution due to $\mathrm{CPO}$, which are induced by the modulation of the optical intensity: 
(a) Slow and fast light device

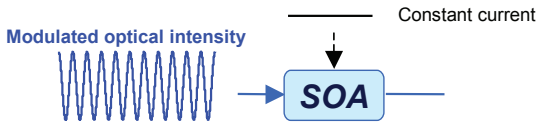

(b) Gain and Phase vs frequency (fixed input power)
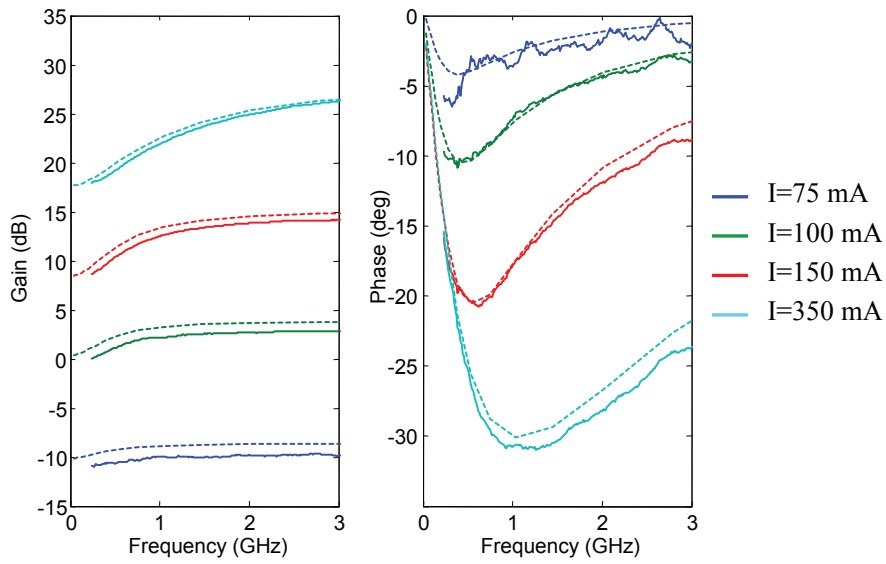

Fig. 4. SOA-based optical delay line. (a) Slow and fast light device. (b) Experimental results: gain and phase shift of the RF signal, at a fixed input optical power $(1 \mathrm{~mW})$, for different currents. Results extracted from (Berger, Alouini, Bourderionnet, Bretenaker \& Dolfi, 2010).

$$
\mathcal{G}^{o p t}=\Gamma \Delta g L M_{0}(0) / M_{1}(0)=\frac{-2 \Gamma g 0 L M_{0} / U_{s}}{1+M_{0} / U_{s}-i \Omega \tau_{s}} .
$$

We represent the module and the argument of the amplification $\mathcal{A}$ and the CPO contribution $\mathcal{G}^{\text {opt }}$ on Fig. 5. The optical amplification $\mathcal{A}$ is in phase with the incident signal, and constant with respect to the RF frequency. At low frequency $\Omega \tau_{s}<1, \mathcal{G}^{\text {opt }}$ is in antiphase with the incident modulated signal. Indeed, the carrier density is modulated by saturation due to the modulated optical intensity: a larger number of carriers will decay near the maximum intensity than near the minimum. Consequently the carrier density is modulated in antiphase with respect to the incident signal, generating a gain also in antiphase. At high frequency $\Omega \tau_{s}>1$, the carriers can no longer follow the optical modulation, and the efficiency of the gain modulation decreases. This explains the low pass filter behavior of $\mathcal{G}^{\text {opt }}$ displayed on Fig. 5. Moreover, in Fig. 5(a), we note that the amplification $\mathcal{A}$ is always dominant compared to $\mathcal{G}^{\text {opt }}$ : consequently at low frequency $\Omega \tau_{s}<1$, the modulated gain $\mathcal{G}^{\text {opt }}$ in antiphase with respect to the incident signal decreases the total gain. This explains the dip at low frequency on the module of the RF transfer function, which is associated with an interesting phase shift, opening the possibility to conceive a tunable delay line.

\subsubsection{Evaluation of the performances for a tunable delay line}

We assume the plane-wave approximation: $M_{1}(z)=\tilde{M}_{1} e^{\tilde{\beta} z}$. Even if it is not truly accurate, it is helpful to understand the essential physics and to evaluate the order of magnitude of 

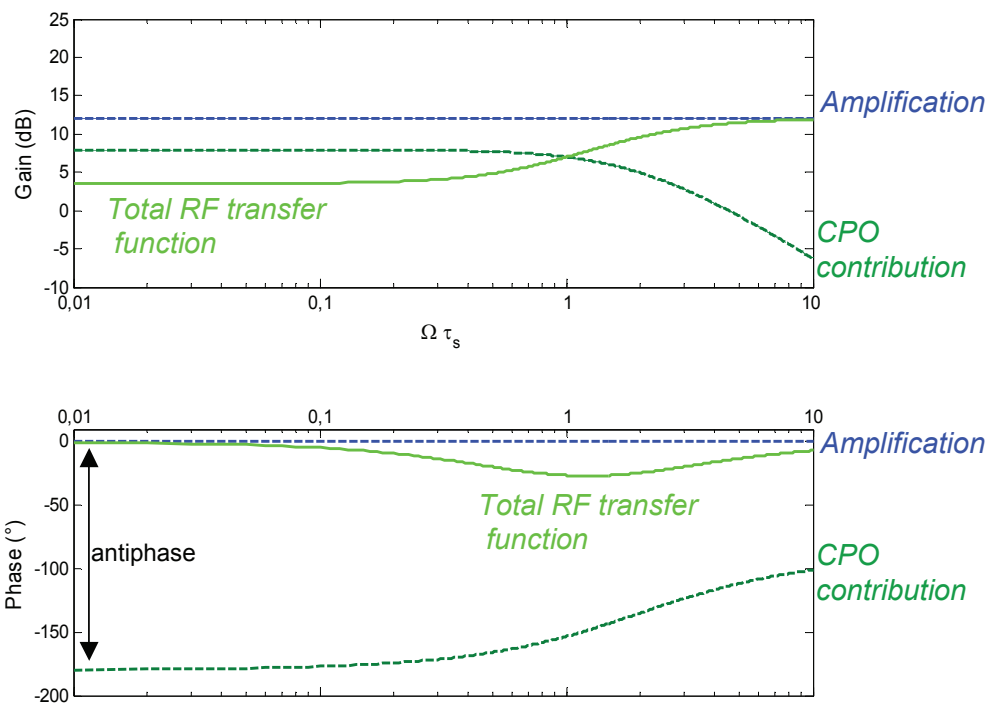

Fig. 5. Contributions and resulting total transfer function.

achievable delay and bandwidth. From Eq. 22, we can deduce the complex propagation constant $\tilde{\beta}$ :

$$
\tilde{\beta} L=-i\left(-\gamma L+\Gamma \bar{g} L-\frac{\Gamma \bar{g} L M_{0} / U_{s}}{1+M_{0} / U_{s}-i \Omega \tau_{s}}\right) .
$$

After a small slice $d z$, if we assume that the gain compensates losses, the output signal is then: $G_{R F} M_{1}(0) e^{-i \Omega(t-\tau)}$, with:

$$
\begin{aligned}
\tau & =-\frac{\Gamma \bar{g} L M_{0} / U_{s}}{\left(1+M_{0} / U_{s}\right)^{2}+\left(\Omega \tau_{s}\right)^{2}} \tau_{s} \\
& \simeq-\frac{\Gamma \bar{g} L M_{0} / U_{s}}{\left(1+M_{0} / U_{s}\right)^{2}} \tau_{s} \text { at low frequency } \Omega \tau_{s}<1+M_{0} / U_{s} .
\end{aligned}
$$

Consequently, at low frequency, CPO introduce a true time advance $\tau$ (negative delay, independent on the frequency $\Omega$ ): Eq. 28 gives the limit of the achievable delay and bandwidth.

It is possible to make a rough evaluation of the maximal achievable bandwidth-delay product, by assuming the saturation parameters $\left(U_{s}, \tau_{s}, \Gamma \bar{g} L\right)$ constant with the current and the input optical power :

$$
\left(\Delta f_{R F} * \tau\right)_{\max }<\frac{1}{2 \pi} \frac{M_{0} / U_{s}}{1+M_{0} / U_{s}}(\Gamma \bar{g} L)_{\max } .
$$

The higher the input optical power $M_{0}$ is, the higher the bandwidth-delay product is. A SOA with a high gain is suitable. However the maximal advance (negative delay) achievable for a given current is: $\tau_{M}(I)=-\frac{1}{4} \Gamma \bar{g} L \tau_{s}$ for a strong input optical power $M_{0} / U_{S} \sim 1$. The delays are tunable from 0 to $\tau_{M}\left(I_{\max }\right)(<0)$, as the gain $\bar{g} L$ governed by the injected current $I$. The delay is null at the transparency, and can be positive when the SOA is in the absorption regime (but it is associated with high losses on the RF signal). In order to refine the performances, and take into account saturation effects, we use the mean saturation 
parameters $\left(U_{s}, \tau_{s}, \Gamma \bar{g} L\right)$ along the SOA, calculated from the model presented in section 4, for a given current and input optical power. For example, a commercial SOA (InP/InGaAsP Quantum Well Booster Amplifier from COVEGA) has a maximal tunable advance of $\approx 516 \mathrm{ps}$ $(\approx 120 \mathrm{ps})$ over an instantaneous bandwidth $<590 \mathrm{MHz}(<410 \mathrm{MHz})$ for an input optical power of $20 \mathrm{~mW}(1 \mathrm{~mW})$.

The major axes of research are now to find the best material to increase these performances, and try to find an architecture which enables to translate these characteristics at any operating frequency.

\subsection{CPO enhanced by index-grating coupling}

\subsubsection{Set-up. experimental results and equations}

(a) Slow and fast light device

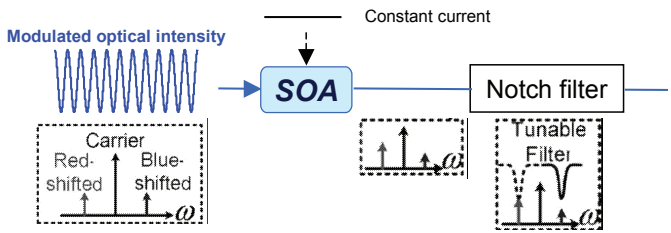

(b) Gain and Phase vs input optical power

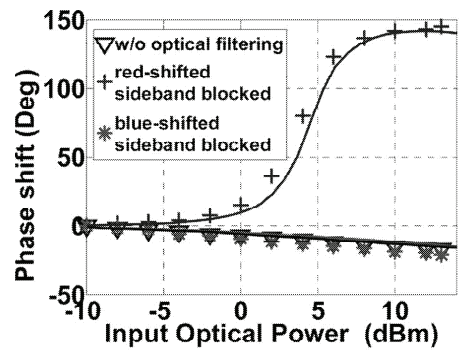

(a)

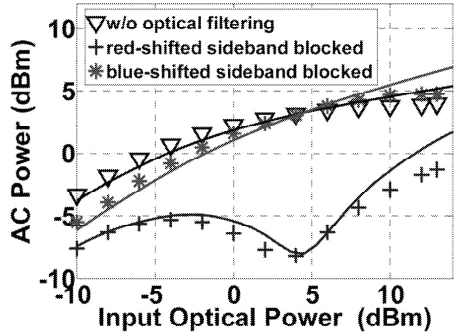

(b)

Fig. 6. CPO enhanced by index-grating coupling. (a) Slow and fast light device. (b) Experimental results: gain and phase shift of the RF signal, at a fixed current $(1 \mathrm{~mW})$, with respect to different input optical power. Graph arranged from (Xue et al., 2008).

As it is explained in the previous paragraph, when both the modulation sidebands are detected by the photodiode, the contribution of the index-gain coupling is canceled out. In order to benefit from the enhancement of the gain and index gratings by the index-gain coupling, Xue et al. (2008) analyze an architecture including an optical notch filter before the photodiode in order to select one sideband (see Fig. 6(a)). They showed that an enhanced phase shift up to $+150^{\circ}$ is detected only when the red shifted sideband is blocked, compared to $-20^{\circ}$ for other configurations (see Fig. 6(b)).

Consequently the RF retrieved signal at the $\Omega$ is either $M_{r}=E_{1} E_{0}^{*}$ when the red-shifted sideband is blocked or $M_{b}=E_{-1} E_{0}^{*}$ when the blue shifted sideband is blocked. Eq. 19 leads to:

$$
\begin{aligned}
& \frac{d M_{r}}{d z}=[-\gamma+\Gamma \bar{g}] M_{r}+\frac{1+i \alpha}{2} \Gamma \Delta g M_{0} \\
& \frac{d M_{b}}{d z}=[-\gamma+\Gamma \bar{g}] M_{b}+\frac{1-i \alpha}{2} \Gamma \Delta g M_{0}
\end{aligned}
$$


with $\Delta g$ defined by Eq. 23 .

\subsubsection{Analysis of the different contributions. Physical interpretation}

We integrate Eq. 30 and Eq. 31 over a small slice $d z$, whose length is noted $L$, to derive an analytical expression of the RF transfer function $S_{21}=\frac{M_{i}(L)}{M_{i}(0)}$ :

$$
\begin{aligned}
& \frac{M_{r}(L)}{M_{r}(0)}=\mathcal{A}+\frac{1}{2} \mathcal{G}^{o p t}+\frac{i \alpha}{2} \mathcal{G}^{o p t}, \\
& \frac{M_{b}(L)}{M_{b}(0)}=\mathcal{A}+\frac{1}{2} \mathcal{G}^{o p t}-\frac{i \alpha}{2} \mathcal{G}^{o p t},
\end{aligned}
$$

with $\mathcal{A}=1-\gamma L+\Gamma \bar{g} L$ and $\frac{1}{2} \mathcal{G}^{\text {opt }}$ expressed as in the previous paragraph (we assume $\left.M_{r}(0)=M_{b}(0)\right)$.

We represent the module and the argument of the amplification $\mathcal{A}$ and the dominant contribution due to CPO: $\pm \frac{i \alpha}{2} \mathcal{G}^{\text {opt }}$ on Fig. 7(a). Contrary to the previous architecture, the contribution enhanced by the gain-index coupling $\pm \frac{i \alpha}{2} \mathcal{G}^{\text {opt }}$, is dominant over the amplification $\mathcal{A}$ at low frequency. At high frequency, the amplification overcomes. Consequently, a transition between the contribution enhanced by the gain-index coupling $\pm \frac{i \alpha}{2} \mathcal{G}^{\text {opt }}$ and the amplification $\mathcal{A}$ is realized at a frequency designed as $f_{t}$. At the transition, the two configurations, when either the red-shifted or blue-shifted sideband is blocked, are not similar: the phase of the enhanced contribution goes from $+90^{\circ}\left(-90^{\circ}\right)$ to $+180^{\circ}\left(0^{\circ}\right)$, when the red (blue)-shifted sideband is blocked. Consequently, if the transition frequency is enough high, when the blue-shifted sideband is blocked, the contribution enhanced by the gain-index coupling $-\frac{i \alpha}{2} \mathcal{G}^{o p t}$ is almost in phase with the amplification $\mathcal{A}$ : the resulting phase of the RF transfer function is continuous at the transition (see Fig. 7(b)). However, when the red-shifted sideband is blocked, the contribution enhanced by the gain-index coupling $+\frac{i \alpha}{2} \mathcal{G}^{\text {opt }}$ is almost in antiphase with the amplification $\mathcal{A}$ : at the transition, the resulting phase of the RF transfer function presents almost a $\pi$ phase jump, associated with a dip in the magnitude of the $\mathrm{RF}$ transfer function. Moreover, the frequency of the transition $f_{t}$ is tunable with either the injected current or the input optical power: consequently at a fixed frequency $f$, the phase of the RF transfer function jump from the phase $\arg \left(+\frac{i \alpha}{2} \mathcal{G}^{\text {opt }}\right) \sim \pi\left(f<f_{t}\right)$ to $\arg (\mathcal{A})=0$ $\left(f>f_{t}\right)$, when the input optical power or the injected current is changed. This interpretation explains well the experimental results.

\subsubsection{Evaluation of the performances for a RF phase shifter}

We have shown in the previous paragraph that the transition is frequency tunable with the injected current or the optical input power. Consequently, for a fixed frequency, it is possible to realize a RF phase shifter. This technique has been applied to realize a tunable opto-electronic oscillator (OEO) (Shumakher et al., 2009a) and a tunable microwave filter (Xue, Sales, Mork \& Capmany, 2009). Due to the involved mechanisms, it is clear that without taken into account propagation effects, the maximal achievable tunable phase shift is $\pi$ per component. A $2 \pi$ phase shift has been realized with cascaded SOAs by Xue, Sales, Capmany \& Mørk (2009). Owing to the high value of the enhancement linewidth factor, the transition frequency $f_{t}$, and then the operating frequency, can be increased up to $\sim 40 \mathrm{GHz}$. 
(a) Contributions
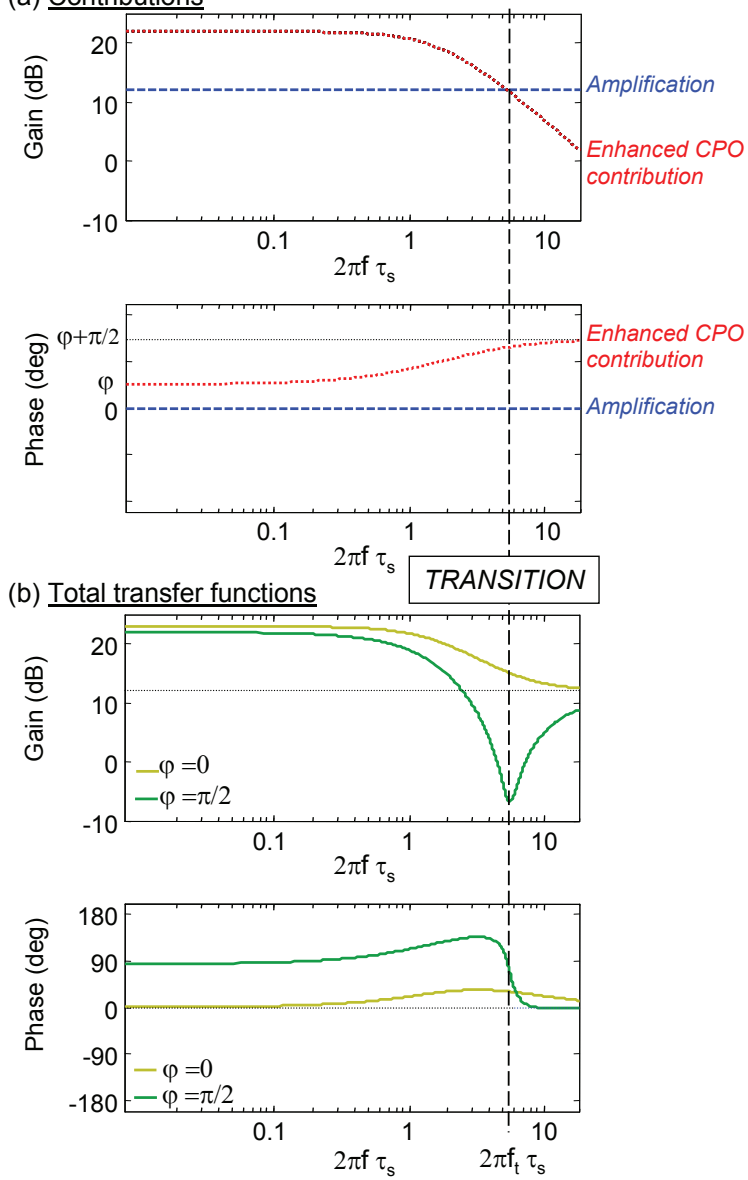

Fig. 7. (a) Contributions to the RF phase change: the red curves correspond both to either the $\mathrm{CPO}$ contribution enhanced by index-grating coupling $\frac{1}{2} \mathcal{G}^{\text {opt }}$ or the CPO contribution enhanced by forced CPO $\frac{1}{2} \mathcal{G}^{\text {elec }}$. When the red or blue shifted sideband is blocked: $\varphi= \pm \pi / 2$; in the case of forced CPO architecture, $\varphi=\phi . \phi$ is the phase difference between the electronic and optical modulations. (b) Total transfer functions obtained from (a), when $\varphi=0$ or $\pi / 2$.

\subsection{Forced CPO in SOA}

We showed in the previous paragraph that the achievable phase shift experienced by an optically carried RF signal passing through the SOA has been recently increased up to $\pi$ by optically filtering out the red-shifted modulation sideband before detection (Xue et al., 2008). However, this method involves the use of a very sharp optical filter and is consequently efficient mainly for relatively high frequencies (typically above $5 \mathrm{GHz}$ ). Furthermore, the insertion of the optical filter leads to a significant enhancement of noise (Shumakher et al., $2009 \mathrm{~b}$ ). Besides, four-wave mixing in SOA have been enhanced by modulating the current of the SOA (Capmany et al., 2002), but the resulting phase shift has never been studied before 
the recent theoretical study by Anton et al. (2009), which points out the enhancement of phase shift by forced CPO. The first experimental observation and a simple physical interpretation of forced CPO is presented in (Berger, Bourderionnet, de Valicourt, Brenot, Dolfi, Bretenaker \& Alouini, 2010).

\subsubsection{Set-up, experimental results and equations}

The slow and fast light device is here a SOA whose current is modulated at the same RF frequency than the input optical power. The input microwave signal modulates then both the injected current of the SOA and the input optical power. An RF attenuator enables to control the modulation depth of the injected current (Fig. 8(a)). The phase difference between the two modulating signals is maintained to $90^{\circ}$. The current of the SOA is set to a fixed current (in the illustration of Fig. 8: $80 \mathrm{~mA}$ ). The gain and the phase shift introduced by the SOA are then measured for different modulation depths of the injected current, by introducing an attenuation $\eta$ on the modulation. The results are shown in Fig. 8(b). Two different regimes can be identified with respect to the frequency. At high frequencies, the response (gain and phase) of the modulated SOA is similar to the response of the non-modulated SOA (usual $\mathrm{CPO}$ behavior, represented by the black dashed curve). On the contrary, at low RF frequencies, forced $\mathrm{CPO}$ occur, and the phase tends to a value between $90^{\circ}$ and $180^{\circ}$ at low frequency. This brings us to define a transition frequency, $f_{t}$, below which the phase of the signal can reach $180^{\circ}$ and above which the phase is close to $0^{\circ}$. These results are then very similar to those obtained by filtering out one of the sideband before the photodiode. Indeed we show in the following paragraph than the physical interpretation is almost the same.

Both the sidebands are detected by the photoreceiver, the equation of propagation is then the same as Eq. 22. However, CPO are not only induced by the modulated optical intensity $M_{0}+M_{1} e^{i \Omega t}+$ c.c., they are also induced by the modulated current $I=I_{0}+\eta I_{1} e^{i \Omega t+\phi}+$ c.c., with $\phi$ the phase shift with respect to the modulation of $M_{0}$. Consequently, $\Delta g$ is expressed as:

$$
\Delta g=\frac{I_{1} e^{i \phi} / I_{s}-\bar{g} M_{1} / U_{s}}{1+M_{0} / U_{s}-i \Omega \tau_{s}}
$$

with $U_{s}=\frac{\hbar \omega}{\Gamma a \tau_{s}}$ and $I_{s}=\frac{q V}{\Gamma a \tau_{s}}$.

\subsubsection{Analysis of the different contributions. Physical interpretation}

We integrate Eq. 22 by taking into account Eq. 34 over a small slice $d z$, whose length is noted $L$, to derive an analytical expression of the RF transfer function $S_{21}=\frac{M_{1}(L)}{M_{1}(0)}$ :

$$
\frac{M_{1}(L)}{M_{1}(0)}=\mathcal{A}+\frac{1}{2} \mathcal{G}^{o p t}+\frac{1}{2} \mathcal{G}^{\text {elec }},
$$

with $\mathcal{A}$ and $\mathcal{G}^{\text {opt }}$ expressed as in the previous paragraphs and $\mathcal{G}^{\text {elec }}$ represents the contribution of forced $\mathrm{CPO}$, generated by the modulated current:

$$
\mathcal{G}^{\text {elec }}=\frac{1}{M_{1}(0)} \frac{I_{1} e^{i \phi} M_{0} / I_{S}}{1+M_{0} / U_{s}-i \Omega \tau_{s}} .
$$

The module and the argument of the amplification term $\mathcal{A}$ and the dominant contribution due to $\mathrm{CPO}: \frac{1}{2} \mathcal{G}^{\text {elec }}$ present the same behavior than in the architecture when one of the sideband is blocked (Fig. 7). The phase of the enhanced contribution goes from $\phi$ to $\phi+90^{\circ}$. Consequently, 
(a) Slow and fast light device

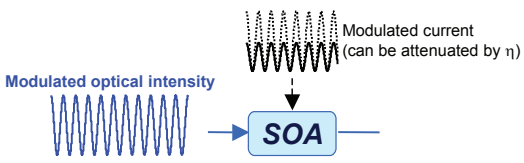

(b) Gain and Phase vs RF frequency

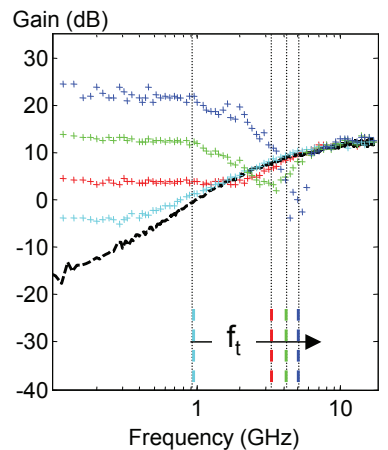

(c) Phase vs modulation depth of the current

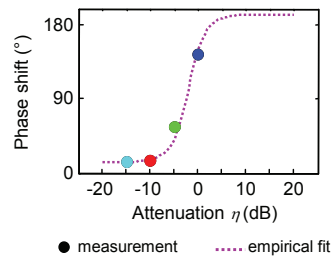

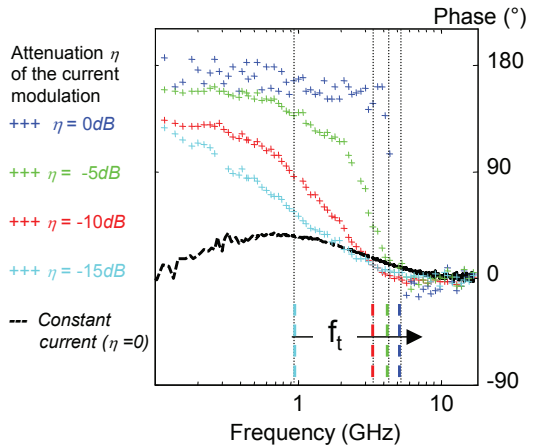

Fig. 8. Forced CPO architecture. (a) Slow and fast light device. (b) Experimental results: gain and phase shift of the RF signal, with respect to the RF frequency, at a fixed current ( $80 \mathrm{~mA})$, and input optical power $(1 \mathrm{~mW})$, for various attenuation of the modulation of the current. (c) RF phase shifter at 3.2 GHz. Graph extracted from (Berger, Bourderionnet, de Valicourt, Brenot, Dolfi, Bretenaker \& Alouini, 2010).

if the phase difference $\phi$ is set to $+90^{\circ}$ (like in the experiment (Berger, Bourderionnet, de Valicourt, Brenot, Dolfi, Bretenaker \& Alouini, 2010)), the configuration is similar to the architecture when the red-shifted sideband is blocked: at the transition between the dominant contribution due to $\mathrm{CPO}$ and the amplification, these latter are almost in antiphase, leading to an almost $\pi$ phase shift and a dip in the gain. The frequency of the transition is tunable with the modulation depth of the current. In Fig. 8(b), we tag the transition frequency $f_{t}$ for each attenuation.

\subsubsection{Evaluation of the performances for a RF phase shifter}

This behavior can be easily exploited to design an adjustable phase shifter, as shown in Fig. 8(c). In this example, the phase is controllable from $15^{\circ}$ to $144^{\circ}$ for a fixed frequency $f=3.2 \mathrm{GHz}$. These performances are similar to those achieved using sideband optical filtering before detection (Xue et al., 2008). In this architecture of forced CPO, a $\pi$ phase shift could be achieved by increasing the current modulation depth. This could also increase the maximum operation frequency of the shifter. Indeed simulations show that a larger ratio between the modulation depth of the current and the optical modulation index would lead to a phase shifter whose operating frequency is close to $10 \mathrm{GHz}$. The main drawback of this architecture is that it involves the use of balanced path for the optical and electronic modulation, and a balanced hybrid $0^{\circ}-90^{\circ}$ power divider, in order to conceive an optically tunable RF phase shifter. 


\section{Generalized CPO model (signal, distortion, noise) based on macroscopic parameters}

In the previous part, we presented the main different architectures published in literature: we explained the essential physics of each of them through a physical interpretation and explained how it can be used as a tunable delay line or a tunable phase shifter. We also roughly evaluate the performances of these architectures as a phase shifter or delay line. Within the scope of integration in a realistic radar system, it is also required to study the impact of these slow and fast light architectures on the performances of the microwave photonics link. In particular, the RF transfer function, the generation of spurious signals by harmonic and intermodulation products, and the intensity noise, have to be studied in order to compute the Spurious Free Dynamic Range (SFDR), a key characteristic in microwave photonics. This part describes the predictive models to compute all of these figure of merits.

\subsection{Model including dynamic saturation}

We derived the equation of propagation in section 2.2 from a phenomenological model of the SOA explained in details by Agrawal \& Dutta (1993). This phenomenological model relies on the assumption that both the variations of the material gain $\Delta g$ and optical index $\Delta n$, caused by a small variation of the carrier density $\Delta N$, are proportional to $\Delta N$. This assumption is based on experimental observations in semiconductor lasers, and is justified in lasers above the threshold: the carrier density $N$ changes little above threshold, and the linear variation is a reasonable approximation for small changes in $N$. Consequently, as far as SOAs are concerned, even if this phenomenological model explains well the essentials physics, as we show in the previous sections, it is not enough for an accurate and predictive model: indeed, this approximation does not give account of strong saturation conditions, with high gain and carrier density variations, which typically occur in quantum wells structures with strong carrier confinement, and can't describe in a predictive way the RF transfer response of the SOA for any operating conditions (injected current, input optical power).

We propose here to describe the model of Berger, Alouini, Bourderionnet, Bretenaker \& Dolfi (2010) which takes into account the carrier density variation along the propagation axis and its influence on the differential gain $a$ and the carrier lifetime $\tau_{s}$. We chose to detail it because it relies on key ideas which makes the predictability and the accuracy of this model, but also of the following models about the distortion and the noise.

Our central hypothesis is that the differential gain $a$ and the carrier lifetime $\tau_{s}$ can be determined as functions of the DC component of the optical intensity $M_{0}$ solely, allowing these dependencies to be determined from gain measurements.

Let us first suppose that we fulfill the small signal condition. In this case, the stimulated emission is negligible compared to the spontaneous emission, leading to the unsaturated steady state solution of the rate equation Eq. 11:

$$
\frac{I}{q L S_{a c t}}=\frac{\bar{N}}{\tau_{s}}
$$

where $L$ is the length of the SOA, $S_{\text {act }}$ is the area of the active section of the SOA. Moreover, we also suppose in this case that the carrier density $\bar{N}$ is constant along the SOA. These hypothesis are justified in moderate length SOAs because the amplified spontaneous emission does not saturate the gain. 
Under these conditions, a measurement of the small signal modal gain $\Gamma g_{0}$ versus $I$ will be equivalent, owing to Eq. 44 , to a determination of the modal gain $\Gamma \bar{g}$ versus $\bar{N} / \tau_{s}$. Here, $\Gamma$ is the ratio $S_{a c t} / S$ of the active to modal gain areas in the SOA.

A last relationship between $\frac{\bar{N}}{\tau_{s}}$ and $M_{0}$ is then required to determine the modal gain $\Gamma \bar{g}$ as a function of $M_{0}$. It is obtained by substituting $\Gamma g\left(\frac{\bar{N}}{\tau_{s}}\right)$ in the saturated steady state solution of the carriers rate equation Eq. 11:

$$
\frac{I}{q L S_{a c t}}-\frac{\bar{N}}{\tau_{s}}-\frac{\Gamma \bar{g}\left(\frac{\bar{N}}{\tau_{s}}\right)}{\hbar \omega} \frac{M_{0}}{\Gamma}=0,
$$

where the injected current $I$ is now fixed by the operating conditions.

Added to the previous relationship between $\Gamma \bar{g}$ and $\frac{\bar{N}}{\tau_{s}}$, the Eq. 45 gives another expression of $\Gamma \bar{g}$ as a function of $\frac{\bar{N}}{\tau_{s}}, \frac{M_{0}}{\Gamma}$ and $I$. Consequently, $\Gamma \bar{g}$ and $\frac{\bar{N}}{\tau_{s}}$ can be known with respect to the local intensity $\frac{M_{0}(z)}{\Gamma}$ and the injected current $I$.

To solve Eqs. 19, we need to express $\bar{N}$ as a function of $\frac{M_{0}(z)}{\Gamma}$ and $I$. This is equivalent to express $\bar{N}$ with respect to $\frac{\bar{N}}{\tau_{s}}$ since $\frac{\bar{N}}{\tau_{s}}$ is known as a function of $\frac{M_{0}(z)}{\Gamma}$ and $I$. Consequently, we model our SOA using the well-known equation:

$$
\frac{\bar{N}}{\tau_{s}}=A \bar{N}+B \bar{N}^{2}+C \bar{N}^{3},
$$

where $A, B$, and $C$, which are respectively the non-radiative, spontaneous and Auger recombination coefficients, are the only parameters that will have to be fitted from the experimental results.

Using Eq. 39 and the fact that we have proved that $\bar{N} / \tau_{s}$ and $\Gamma \bar{g}$ can be considered as function of $\frac{M_{0}(z)}{\Gamma}$ and $I$ only, we see that $\bar{N}, \Gamma a=\Gamma \frac{\partial \bar{g}}{\partial N}$, and $\frac{U_{s}}{\Gamma}=\frac{\hbar \omega}{\Gamma a \tau_{s}}$ can also be considered as functions of $\frac{M_{0}(z)}{\Gamma}$ and $I$. This permits to replace Eqs. 19 by the following system:

$$
\begin{aligned}
\frac{d M_{0}}{d z} & =\left(\Gamma \bar{g}\left(\frac{M_{0}(z)}{\Gamma}, I\right)-\gamma\right) M_{0} \\
\frac{d E_{1}}{d z} & =\frac{1}{2}\left(\Gamma \bar{g}\left(\frac{M_{0}(z)}{\Gamma}, I\right)-\gamma\right) E_{1}+\frac{1-i \alpha}{2} \Gamma \Delta g\left(\frac{M_{0}(z)}{\Gamma}, I\right) E_{0} \\
\frac{d E_{-1}^{*}}{d z} & =\frac{1}{2}\left(\bar{g}\left(\frac{M_{0}(z)}{\Gamma}, I\right)-\gamma\right) E_{-1}^{*}+\frac{1+i \alpha}{2} \Gamma \Delta g\left(\frac{M_{0}(z)}{\Gamma}, I\right) E_{0}^{*},
\end{aligned}
$$

with:

$$
\Delta g\left(\frac{M_{0}(z)}{\Gamma}, I\right)=\frac{M_{1} / U_{s}\left(\frac{M_{0}(z)}{\Gamma}, I\right)}{1+\Gamma M_{0} / U_{s}\left(\frac{M_{0}(z)}{\Gamma}, I\right)-i \Omega \tau_{s}\left(\frac{M_{0}(z)}{\Gamma}, I\right)}
$$

Eqs. 40, 41 and 42 are then numerically solved: Eq. 40 gives $\frac{M_{0}(z)}{\Gamma}$, with the initial condition $\frac{M_{0}(0)}{\Gamma}=\sqrt{\gamma} \frac{P_{i n}}{S_{a c t}}$, where $P_{\text {in }}$ is the optical input power. $\frac{M_{0}(z)}{\Gamma}$ can be then introduced into Eqs. 41, 42. It is then possible to simulate the optical fields $E_{1}, E_{-1}$, or the RF signal $M_{1}$ (which is equal either to $E_{0}^{*} E_{1}+E_{0} E_{-1}^{*}$, or to $E_{0}^{*} E_{1}$, or to $E_{0} E_{-1}^{*}$, depending on the modulation format before the photodiode).

It is important to note that the recombination coefficients $A, B$ and $C$ are the only fitting parameters of this model. Once obtained from experimental data, they are fixed for any other 
experimental conditions. Moreover, the only geometrical parameters that are required are the length $L$ of the SOA and the active area cross section $S_{a c t}$. The derivation of a predictive model, independent of the experimental conditions (current and input optical power) is then possible, provided that the simple measurements of the total losses and the small signal gain versus the current are conducted. The above model lies in the fact that first, the spatial variations of the saturation parameters are taken into account, and second, their values with respect to the local optical power are deduced from a simple measurement. These keys ideas lead to a very convenient model of the microwave complex transfer function of the SOA, and then of the slow light properties of the component. It can be easily used to characterize commercial components whose design details are usually unknown. We illustrate the accuracy and the robustness of the model in the part 5. Lastly, it is worth mentioning that in order to compute the complex transfer function of an architecture including a SOA and a filter, the complex transfer function of the filter has to be then applied to the output field compounds $E_{k}$ computed by the previous model (Dúill et al., 2010a).

\subsection{Distortion model}

The model we present in this part is a generalization of the former one. It enables to take into account higher order coherent population oscillations due to large signal modulation, or the non-linearities at the input of the SOA (from the Mach-Zehnder that modulates the optical beam for example), and can be used to compute the harmonic generation and the intermodulation products. The detailed model is presented in (Berger, Bourderionnet, Alouini, Bretenaker \& Dolfi, 2009).

\subsubsection{Harmonic generation}

In order to find the level of the generated harmonics, we first consider that the input optical field is modulated at the RF frequency $\Omega .|E|^{2}, g$ and $N$ are hence all time-periodic functions with a fundamental frequency of $\Omega$. They can therefore be written into Fourier harmonic decompositions:

$$
\begin{aligned}
|E(z, t)|^{2} & =\sum_{k=-\infty}^{+\infty} M_{k}(z) e^{-i k \Omega t}, \\
N(z, t) & =\bar{N}(z)+\sum_{\substack{k=-\infty \\
k \neq 0}}^{+\infty} N_{k}(z) e^{-i k \Omega t}, \\
g(z, t) & =\bar{g}(z)+a(z) \sum_{\substack{k=-\infty \\
k \neq 0}}^{+\infty} N_{k}(z) e^{-i k \Omega t}
\end{aligned}
$$

where $\bar{N}(z)$ and $\bar{g}(z)$ respectively denote the DC components of the carrier density and of the optical gain. $a(z)$ is the SOA differential gain, defined as $a(z)=\partial \bar{g} / \partial \bar{N}$. Defining $g_{k}$ as the oscillating component of the gain at frequency $k \Omega$, and considering only a finite number $K$ of harmonics, the carrier rate equation (Eq. 11) can be written as:

$$
\hbar \omega\left(\frac{I}{q V}-\frac{\bar{N}}{\tau_{s}}\right)=\alpha_{0} \bar{g}+\sum_{\substack{p+q=0 \\ p, q \in[-K, K] \\ p \neq 0}} g_{p} M_{q},
$$




$$
0=\alpha_{i} g_{i}+\sum_{\substack{p+k=i \\ p, q \in[-K, K] \\ p \neq i}} g_{p} M_{q}, \text { for } i \neq 0 \text { and } i \in[-K, K]
$$

where $\alpha_{k}=U_{s}\left(1+M_{0} / I_{s}-i k \Omega \tau_{s}\right)$, and $\alpha_{0}=M_{0}$ is the DC optical intensity. $U_{s}$ denotes the local saturation intensity and is defined as $U_{s}=\hbar \omega / a \tau_{s}$. It is worth mentioning that $\alpha_{k}$ is obtained at the first order of equation (Eq. 11), when mixing terms are not considered. It is important to note that in the following, $\bar{N}, \bar{g}, a, \tau_{s}, U_{s}$, and consequently the $\alpha_{k}$ 's are all actually functions of $z$. Their variations along the propagation axis is then taken into account, unlike most of the reported models in which effective parameters are used (Agrawal, 1988; Mørk et al., 2005; Su \& Chuang, 2006).

In order to preserve the predictability of the model, $\bar{g}, U_{s}$ and $\tau_{s}$ has to be obtained as in the small signal case. However, in the case of a large modulation index, an iterative procedure has to be used: in a first step, we substitute $\bar{N} / \tau_{s}, U_{S}$ and $\tau_{s}$ in (47) by their small signal values $\bar{N} / \tau_{s}^{(0)}, U_{s}^{(0)}$ and $\tau_{s}^{(0)}$. The gain components $\bar{g}$ and $g_{k}$ can be then extracted from Eqs. 47 and 48. Similarly to the small signal case, using equations (39) and (47), we obtain $\bar{N} / \tau_{s}^{(1)}, U_{s}^{(1)}$ and $\tau_{s}^{(1)}$ as functions of $I, A, B, C$ and $M_{k}(z)$. This procedure is repeated until convergence of $\bar{N} / \tau_{s}^{(n)}, U_{s}^{(n)}$ and $\tau_{s}^{(n)}$, which typically occurs after a few tens of iterations.

The propagation equation (Eq 19) can now be expressed as:

$$
\frac{d E_{k}}{d z}=\frac{1}{2}\left(\bar{g}-\gamma_{i}\right) E_{k}+\frac{1-i \alpha}{2} \sum_{\substack{p+q=k,-K<p, q<K}} \Gamma g_{p} E_{q},
$$

From these equations it is straightforward to deduce the equation for the component $M_{k}$ of the optical intensity, either if the modulation is single-sideband or double-sideband. For numerical simulations, it is very useful to express the Eqs. 47, 48 and 49 in a matrix formulation. The expressions can be found in (Berger, Bourderionnet, Alouini, Bretenaker \& Dolfi, 2009).

In the case of a real microwave photonics link, the harmonics at the input of the SOA, created by the modulator, has to be taken into account. By using the reported model, the third harmonic photodetected power, can be evaluated with:

$$
H_{3}=2 R \eta_{p h}^{2}\left|M_{3, \text { out }} \times S\right|^{2}
$$

where $\mathrm{R}$ and $\eta_{p h}$ are respectively the photodiode resistive load (usually $50 \Omega$ ) and efficiency (assumed to be equal to 1). $S$ denotes the SOA modal area.

\subsubsection{Intermodulation distortion}

Intermodulation distortion (IMD) calculation is slightly different from what has been discussed in the above section. Indeed, the number of mixing terms that must be taken into account is significantly higher. For radar applications a typical situation where the IMD plays a crucial role is that of a radar emitting at a RF frequency $\Omega_{1}$, and facing a jammer emitting at $\Omega_{2}$, close to $\Omega_{1}$. Both $\Omega_{1}$ and $\Omega_{2}$ are collected by the antenna and transferred to the optical carrier through a single electro-optic modulator. The point is then to determine the nonlinear frequency mixing due to the CPO inside the SOA. In particular, the mixing products at frequencies $\Omega_{2}-\Omega_{1}$ (or $\left.\Omega_{1}-\Omega_{2}\right)$ and $2 \Omega_{2}-\Omega_{1}\left(\right.$ or $\left.2 \Omega_{1}-\Omega_{2}\right)$ - respectively called second 
$\left(\mathrm{IMD}_{2}\right)$ and third $\left(\mathrm{IMD}_{3}\right)$ order intermodulation distortions - have to be evaluated at the output of the SOA.

The main difference with harmonic calculation is that the optical intensity, and hence the SOA carrier density $N$, and the SOA gain $g$ are no longer time-periodic functions of period $\Omega$, but also of period $\delta \Omega=\Omega_{2}-\Omega_{1}$.

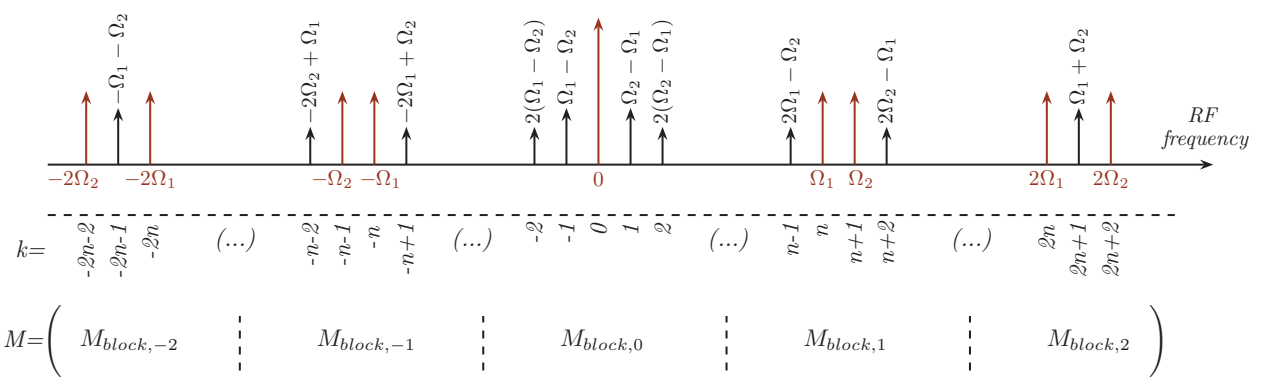

Fig. 9. Set of significant spectral components of $|E|^{2}, N$ and $g$, and associated index $k$ in their Fourier decompositions. $n$ is defined such as $\Omega_{1}=n \delta \Omega$. Graph extracted from (Berger, Bourderionnet, Alouini, Bretenaker \& Dolfi, 2009).

We consider a typical radar frequency $\Omega_{1}$ of $10 \mathrm{GHz}$, and a frequency spacing $\delta \Omega$ of $10 \mathrm{MHz}$. Here, for intermodulation distortion calculation, we assume that only the spectral components at $\Omega_{1,2}, 2 \Omega_{1,2}$, and all their first order mixing products significantly contribute to the generation of $\mathrm{IMD}_{2}$ and $\mathrm{IMD}_{3}$, as illustrated in figure 9. The $M_{k}{ }^{\prime} \mathrm{s}$ and the $g_{k}$ 's are then reduced in 19 elements vectors which can be gathered into blocks, the $j^{\text {th }}$ block containing the mixing products with frequencies close to $j \times \Omega_{1}$. The Eqs. 47,48 and 49 can be then be written as matrices in block, and the full procedure described in the previous can be applied in the same iterative way to determine the $g_{k}{ }^{\prime} s, U_{s}$ and $\tau_{s}$, and to finally numerically solve the equation (49). Detailed matrices are presented in (Berger, Bourderionnet, Alouini, Bretenaker \& Dolfi, 2009). Similarly to equation (50), the photodetected RF power at $2 \Omega_{2}-\Omega_{1}$ is then calculated through:

$$
\mathrm{IMD}_{3}=2 R \eta_{p h}^{2}\left|M_{2 \Omega_{2}-\Omega_{1}}^{\text {out }} \times S\right|^{2} .
$$

We explained in this section how to adapt the predictive small-signal model including dynamic saturation, in order to compute the harmonics and the intermodulation products, while keeping the accuracy and predictability of the model. It is worth noticing that in a general way, the propagation of the Fourier compounds of an optically carried microwave signal into the SOA can be seen as resulting from an amplification process and a generation process by frequency mixing through CPO. We will see in part 5 how these two effects, which are in antiphase, can be advantageously used to linearize a microwave photonics link.

In order to compute the dynamic range of a microwave photonics link, the only missing characteristic is the intensity noise.

\subsection{Intensity noise}

The additional intensity noise can be extracted from the model of the RF transfer function described in section 4.1. The principle is detailed in (Berger, Alouini, Bourderionnet, Bretenaker \& Dolfi, 2009b). Indeed, when the noise is described in the semi-classical beating 
theory, the fields contributing to the intensity noise are the optical carrier and the spontaneous emission. We define the input spontaneous emission power density as the quantum noise source at the input of SOA, which can be extracted from a measurement of the optical noise factor. The input intensity is then composed of:

(1) a spontaneous-spontaneous beat-note which is only responsive to the optical gain.

(2) a carrier-spontaneous beat-note, which can be considered as an optical carrier and a sum of double-sideband modulation components at the frequency $\Omega$ (Olsson, 1989). However, the right-shifted and the blue-shifted sidebands at $\Omega$ are incoherent. Consequently, the double sidebands at $\Omega$ has to be taken into account as two independent single-sideband modulations. Their respective contributions to the output intensity noise can be then computed from the model of the RF transfer function described in section 4.1. All the contributions are finally incoherently summed.

The relative intensity noise and the noise spectral density can be then easily modeled from the RF transfer function described in section 4.1. It is interesting to observe that first this model leads to an accurate description of the output intensity noise (Berger, Alouini, Bourderionnet, Bretenaker \& Dolfi, 2009b). Secondly, we can show that the relative intensity noise after a SOA (without optical filter) is proportional to the RF transfer function, leading to an almost constant carrier-to-noise ratio with respect to the RF frequency (Berger, Alouini, Bourderionnet, Bretenaker \& Dolfi, 2009a): the dip in the gain associated to tunable delays, does not degrade the carrier-to-noise ratio. However, it is not anymore valid when an optical filter is added before the photodiode (Duill et al., 2010b; Lloret et al., 2010), due to the incoherent sum of the different noise contributions.

\section{Dynamic range of slow and fast light based SOA link, used as a phase shifter}

We focus here on the study of a single stage phase shifter consisting of a SOA followed by an optical notch filter (ONF), which attenuates the red shifted modulation sideband (see section 3.2). In order to be integrated in a real radar system, the influence of such an architecture on the microwave photonics link dynamic range has to be studied. The large phase shift obtained by red sideband filtering is however accompanied by a significant amplitude reduction of the RF signal at the phase jump. An important issue in evaluating the merits of the filtering approach is its effect on the linearity of the link. Indeed, similarly to the fundamental signal whose characteristics evolve with the degree of filtering, it is expected that attenuating the red part of the spectrum should affect the nonlinear behavior of the CPO based phase shifter. The nonlinearity we consider here is the third order intermodulation product (IMD3). This nonlinearity accounts for the nonlinear mixing between neighboring frequencies $f_{1}$ and $f_{2}$ of the RF spectrum, and refers to the detected RF power at frequencies $2 f_{2}-f_{1}$ and $2 f_{1}-f_{2}$. Since these two frequencies are close to $f_{1}$ and $f_{2}$, this quantity is of particular importance in radar and analog transmission applications, where IMD3 is the dominant detrimental effect for MWP links (Ackerman, 1994).

To this aim, the predictions of the model presented in the previous part are compared with experimental results (RF complex transfer function, intermodulation products IMD3). Then we use our predictive model to find out the guidelines to optimize a microwave photonics link including a SOA based phase shifter.

\subsection{Experimental confirmation of the model predictions}

The experimental set-up for IMD3 measurement is depicted on Fig. 10. The RF tones are generated by two RF synthesizers at $f_{1}=10 \mathrm{GHz}$ and $f_{2}=10.01 \mathrm{GHz}$. The two RF signals are 


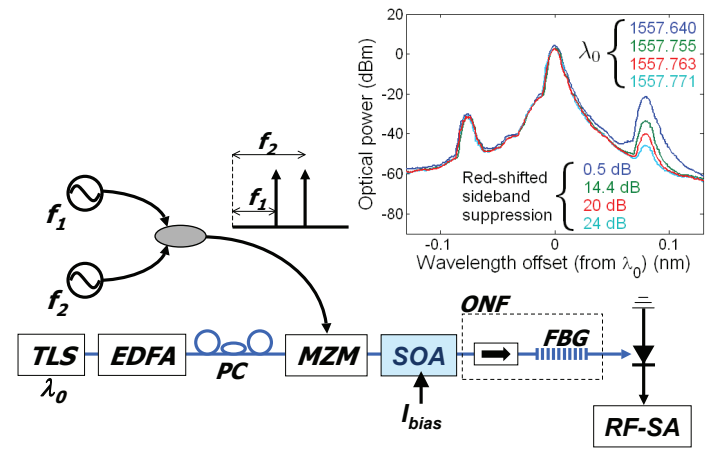

Fig. 10. Experimental setup for IMD3 measurement. EDFA, Erbium-Doped Fiber Amplifier; $\mathrm{PC}$, Polarization Controller. The red-shifted sideband attenuation is varied from $0.5 \mathrm{~dB}$ to $24 \mathrm{~dB}$ (inset). Extracted from (Berger, Bourderionnet, Bretenaker, Dolfi, Dúill, Eisenstein \& Alouini, 2010).

combined to drive a zero-chirp Mach-Zehnder modulator (MZM). A single frequency tunable laser source (TLS) feeds the MZM. The optically carried signal is coupled to the phase shifting element that comprises a commercial SOA, an optical notch filter made of an isolator, and a fiber Bragg grating (FBG). The $3 \mathrm{~dB}$ spectral bandwidth of the FBG is $0.2 \mathrm{~nm}$. The SOA gain and the output saturation power are respectively $21.3 \mathrm{~dB}$ and $15 \mathrm{dBm}$ at $500 \mathrm{~mA}$ bias current. The optical power at the SOA input was set to $10 \mathrm{dBm}$ which ensures operation in strong saturation conditions, which is favorable for phase shifting applications (Shumakher et al., 2009b). The various degrees of red-shifted optical sideband filtering are obtained by tuning the optical carrier wavelength with respect to the central wavelength of the notch filter (see Fig. 10, inset). Finally, to get rid of all photodiode nonlinearities, an optical attenuator was placed before the detector. The detected RF signal is then sent to a RF spectrum analyzer which records the output RF power at the four frequencies $f_{1}, f_{2}, 2 f_{2}-f_{1}$ and $2 f_{1}-f_{2}$ for IMD3 evaluation. The RF phase shift at $10 \mathrm{GHz}$ is also measured using a vector network analyzer (VNA). For our measurements, we considered four different wavelengths, that is, four degrees of optical filtering (see inset of Fig. 10).

The plots presented in Fig. 11 correspond respectively from left to right to a red-shifted sideband optical attenuation of $0.5,14.4,20$ and $24 \mathrm{~dB}$. The model predictions are in very good agreement with the experimental results, for the RF phase, the RF output power and the output IMD3 power.

The most important result of Fig. 11 is that theory and experiment agree remarkably well to evidence the presence of a dip in power-versus-current characteristics of both the RF signal and the IDM3. Although the two dips do not occur at the same bias current (respectively $200 \mathrm{~mA}$ and $120 \mathrm{~mA}$ for fundamental RF signal and IMD3), they behave similarly with respect to the degree of filtering. Both dips decrease as the red-shifted sideband filtering diminishes, and completely disappear in the absence of filtering. Indeed, as we explained in section, the filtering reveals the index-gain coupling. Near the dip, the output signal comes equally from the amplification of the input signal (contribution in phase), and from CPO enhanced by index-coupling, (contribution almost in antiphase at $10 \mathrm{GHz}$ ) (see Fig. 7).

However, the dip in the IMD3 doesn't lead directly to an improvement of the dynamic range as it doesn't appear for the same operating conditions than the phase shift. A trade-off has to be found between the available phase shift and the dynamic range. 

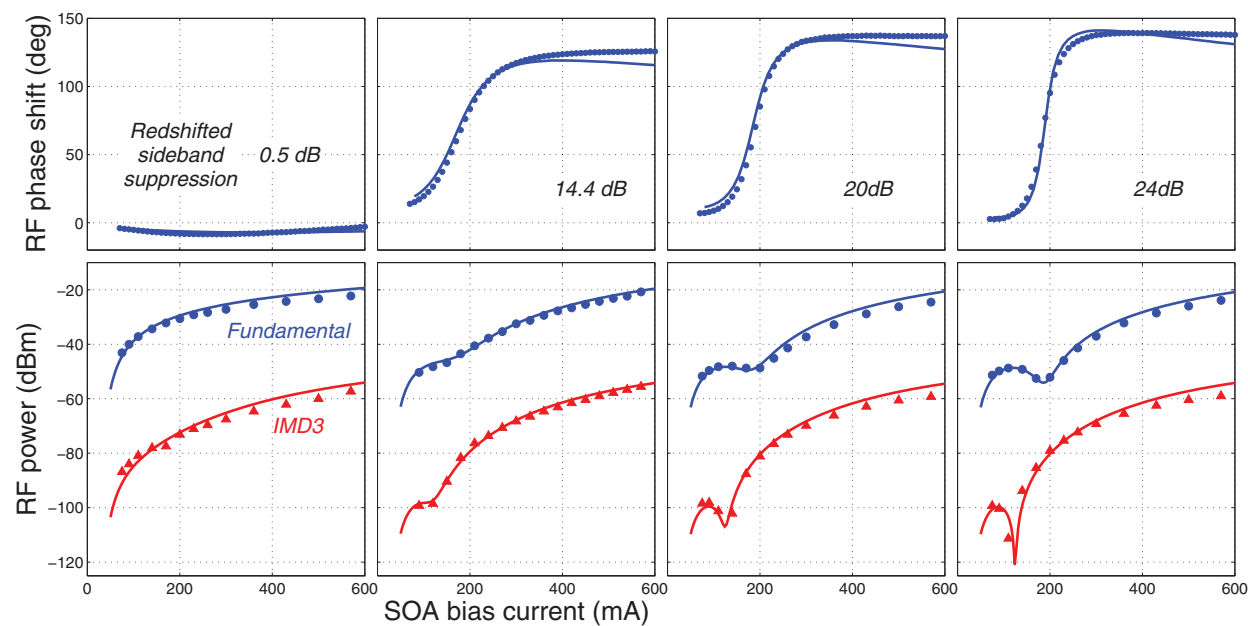

Fig. 11. Top: RF phase shift at $10 \mathrm{GHz}$ versus SOA bias current; Bottom: RF power at fundamental frequency $f_{1}$ (in blue), and at $2 f_{2}-f_{1}$, (IMD3, in red). From left to right, red-shifted sideband attenuation increases from $0.5 \mathrm{~dB}$ to $24 \mathrm{~dB}$. Symbols represent experimental measurements, and solid lines show theoretical calculations. Extracted from (Berger, Bourderionnet, Bretenaker, Dolfi, Dúill, Eisenstein \& Alouini, 2010).

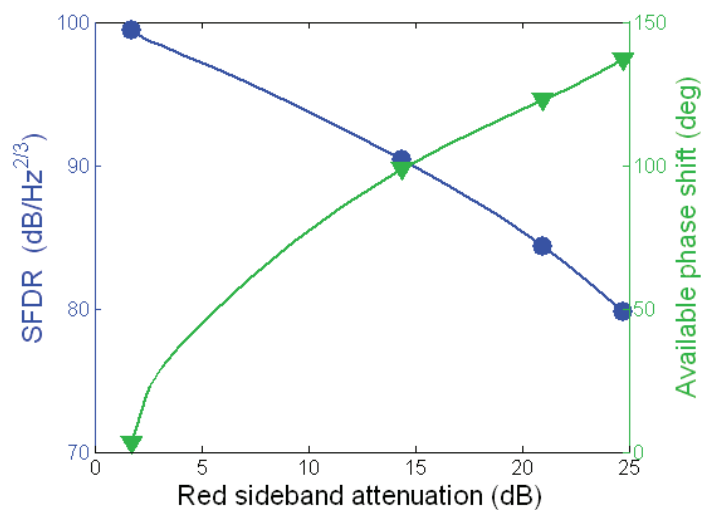

Fig. 12. In blue: Spurious Free Dynamic Range (SFDR); in green: available phase shift. Both are represented with respect to the red sideband attenuation. The model prediction is represented by a line, the dots are the experimental points. 


\subsection{Influence of the optical filtering on the performance of the phase shifter}

To this aim, we compute the Spurious-Free Dynamic Range (SFDR), which is the key figure of the dynamic range in microwave photonics (Ackerman, 1994). It is defined as the RF power range where the intermodulation products IMD3 are below the noise floor. We represent in Fig. 12 the SFDR and the available phase shift with respect to the red sideband attenuation. It appears that the best trade-off between the dynamic range and the available phase shift corresponds to the minimum strength of filtering which enables to reveal the index-gain coupling. With this non-optimized link, we reach a SFDR of $90 \mathrm{~dB} / \mathrm{Hz}^{2 / 3}$ for an available phase shift of 100 degrees.

\subsection{Linearized amplification at high frequency}

In a more general context, a SOA can be used to reduces the non-linearities of a microwave photonics link. Indeed, the input linearities (from the modulator for example) can be reduced by the nonlinearities generated by the gain in antiphase created by the CPO. It has already been demonstrated using a single SOA (without optical filter) at low frequency ( $2 \mathrm{GHz}$ ) (Jeon et al., 2002)). However with a single SOA, the gain in antiphase due to CPO is created only at low frequency (below a few GHz), as it is illustrated on Fig. 5. However, when the SOA is followed by an optical filter attenuating the red-shifted sideband, the gain in antiphase is created at high frequency, as it is illustrated on Fig. 7. This architecture enables then a linearization of the microwave photonics link well beyond the inverse of the carrier lifetime. Indeed we have experimentally demonstrated that a dip in the IMD3 occurs at $10 \mathrm{GHz}$ (Fig. 11). However the instantaneous bandwidth is still limited to the GHz range.

\section{Conclusion}

We have reviewed the different set-ups proposed in literature, and we have given the physical interpretation of each architecture, aiming at helping the reader to understand the underlying physical mechanisms.

Moreover, we have shown that a robust and predictive model can be derived in order to simulate and understand the RF transfer function, the generation of spurious signals through harmonic distortion and intermodulation products, and the intensity noise at the output of a SOA. This model takes into account the dynamic saturation along the propagation in the SOA, which can be fully characterized by a simple measurement, and only relies on material fitting parameters, independent of the optical intensity and the injected current. In these conditions, the model is found to be predictive and can be used to simulate commercial SOAs as well. Moreover, we have presented a generalization of the previous model, which permits to describe harmonic generation and intermodulation distortions in SOAs. This model uses a rigorous expression of the gain harmonics. Lastly, we showed the possibility to use this generalized model of the RF transfer function to describe the intensity noise at the output of the SOA.

This useful tool enables to optimize a microwave photonics link including a SOA, by finding the best operating conditions according to the application. To illustrate this point, the model is used to find out the guidelines for improving the MWP link dynamic range using a SOA followed by an optical filter, in two cases: first, for phase shifting applications, we have shown that the best trade-off between the dynamic range and the available phase shift corresponds to the minimum strength of filtering which enables to reveal the index-gain coupling. Second, we have experimentally demonstrated and have theoretically explained how an architecture 
composed of a SOA followed by an optical filter can reduce the non-linearities of the modulator, at high frequency, namely beyond the inverse of the carrier lifetime.

\section{References}

Ackerman, E. (1994). Photonic Aspects of modern radar, Artech House.

Agrawal, G. P. (1988). Population pulsations and nondegenerate four-wave mixing in semiconductor lasers and amplifiers, J. Opt. Soc. Am. B 5(1): 147-159.

Agrawal, G. P. \& Dutta, N. K. (1993). Semiconductor Lasers, Kluwer Academic, Boston.

Anton, M. A., Carreno, F., Calderon, O. G., Melle, S. \& Arrieta-Yanez, F. (2009). Phase-controlled slow and fast light in current-modulated SOA, Journal of Physics B: Atomic, Molecular and Optical Physics 42(9): 095403 (8pp).

Berger, P., Alouini, M., Bourderionnet, J., Bretenaker, F. \& Dolfi, D. (2009a). Influence of slow light effect in semiconductor amplifiers on the dynamic range of microwave-photonics links, Slow and Fast Light, Optical Society of America, p. SMB6.

Berger, P., Alouini, M., Bourderionnet, J., Bretenaker, F. \& Dolfi, D. (2009b). Slow light using semiconductor optical amplifiers: Model and noise characteristics, C. R. Physique 10: 991-999.

Berger, P., Alouini, M., Bourderionnet, J., Bretenaker, F. \& Dolfi, D. (2010). Dynamic saturation in semiconductoroptical amplifiers: accurate model, roleof carrier density, and slow light, Opt. Express 18(2): 685-693.

Berger, P., Bourderionnet, J., Alouini, M., Bretenaker, F. \& Dolfi, D. (2009). Theoretical study of the spurious-free dynamic range of a tunable delay line based on slow light in soa, Opt. Express 17(22): 20584-20597.

Berger, P., Bourderionnet, J., Bretenaker, F., Dolfi, D., Dúill, S. O., Eisenstein, G. \& Alouini, M. (2010). Intermodulation distortion in microwave phase shifters based on slow and fast light propagation in SOA, Opt. Lett. 35(16): 2762-2764.

Berger, P., Bourderionnet, J., de Valicourt, G., Brenot, R., Dolfi, D., Bretenaker, F. \& Alouini, M. (2010). Experimental demonstration of enhanced slow and fast light by forced coherent population oscillations in a semiconductor optical amplifier, Opt. Lett. 35: 2457.

Bogatov, A. P., Eliseev, P. G. \& Sverdlov, B. N. (1975). Anomalous interaction of spectral modes in a semiconductor laser, Quantum Electronics, IEEE Journal of 11: 510.

Boula-Picard, R., Alouini, M., Lopez, J., Vodjdani, N. \& Simon, J.-C. (2005). Impact of the gain saturation dynamics in semiconductor optical amplifiers on the characteristics of an analog optical link,

Capmany, J., Sales, S., Pastor, D. \& Ortega, B. (2002). Optical mixing of microwave signals in a nonlinear semiconductor laser amplifier modulator, Opt. Express 10(3): 183-189.

Dúill, S. O., Shumakher, E. \& Eisenstein, G. (2010a). The role of optical filtering in microwave phase shifting, Opt. Lett. 35(13): 2278-2280.

Duill, S., Shumakher, E. \& Eisenstein, G. (2010b). Noise properties of microwave phase shifters based on SOA, Lightwave Technology, Journal of 28(5): 791 -797.

Henry, C. (1982). Theory of the linewidth of semiconductor lasers, Quantum Electronics, IEEE Journal of 18(2): $259-264$.

Jeon, D.-H., Jung, H.-D. \& Han, S.-K. (2002). Mitigation of dispersion-induced effects using soa in analog optical transmission, Photonics Technology Letters, IEEE 14(8): 1166 - 1168. 
Lloret, J., Ramos, F., Sancho, J., Gasulla, I., Sales, S. \& Capmany, J. (2010). Noise spectrum characterization of slow light soa-based microwave photonic phase shifters, Photonics Technology Letters, IEEE 22(13): 1005 -1007.

Mørk, J., Kjær, R., van der Poel, M. \& Yvind, K. (2005). Slow light in a semiconductor waveguide at gigahertz frequencies, Opt. Express 13(20): 8136-8145.

Olsson, N. (1989). Lightwave systems with optical amplifiers, Lightwave Technology, Journal of 7(7): 1071-1082.

Peatross, J., Glasgow, S. A. \& Ware, M. (2000). Average energy flow of optical pulses in dispersive media, Phys. Rev. Lett. 84(11): 2370-2373.

Pesala, B., Chen, Z., Uskov, A. V. \& Chang-Hasnain, C. (2006). Experimental demonstration of slow and superluminal light in semiconductor optical amplifiers, Opt. Express 14(26): 12968-12975.

Shumakher, E., Duill, S. \& Eisenstein, G. (2009a). Optoelectronic oscillator tunable by an soa based slow light element, Lightwave Technology, Journal of 27(18): 4063-4068.

Shumakher, E., Dúill, S. O. \& Eisenstein, G. (2009b). Signal-to-noise ratio of a semiconductor optical-amplifier-based optical phase shifter, Opt. Lett. 34(13): 1940-1942.

$\mathrm{Su}, \mathrm{H}$. \& Chuang, S. L. (2006). Room temperature slow and fast light in quantum-dot semiconductor optical amplifiers, Applied Physics Letters 88(6): 061102.

Xue, W., Chen, Y., Öhman, F., Sales, S. \& Mørk, J. (2008). Enhancing light slow-down in semiconductor optical amplifiers by optical filtering, Opt. Lett. 33(10): 1084-1086.

Xue, W., Sales, S., Capmany, J. \& Mørk, J. (2009). Experimental demonstration of 360otunable rf phase shift using slow and fast light effects, Slow and Fast Light, Optical Society of America, p. SMB6.

Xue, W., Sales, S., Mork, J. \& Capmany, J. (2009). Widely tunable microwave photonic notch filter based on slow and fast light effects, Photonics Technology Letters, IEEE 21(3): 167-169. 


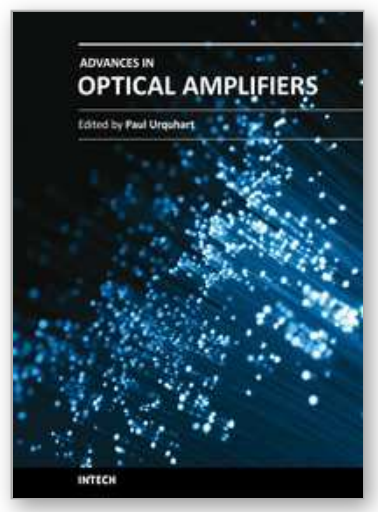

\author{
Advances in Optical Amplifiers \\ Edited by Prof. Paul Urquhart
}

ISBN 978-953-307-186-2

Hard cover, 436 pages

Publisher InTech

Published online 14, February, 2011

Published in print edition February, 2011

Optical amplifiers play a central role in all categories of fibre communications systems and networks. By compensating for the losses exerted by the transmission medium and the components through which the signals pass, they reduce the need for expensive and slow optical-electrical-optical conversion. The photonic gain media, which are normally based on glass- or semiconductor-based waveguides, can amplify many high speed wavelength division multiplexed channels simultaneously. Recent research has also concentrated on wavelength conversion, switching, demultiplexing in the time domain and other enhanced functions. Advances in Optical Amplifiers presents up to date results on amplifier performance, along with explanations of their relevance, from leading researchers in the field. Its chapters cover amplifiers based on rare earth doped fibres and waveguides, stimulated Raman scattering, nonlinear parametric processes and semiconductor media. Wavelength conversion and other enhanced signal processing functions are also considered in depth. This book is targeted at research, development and design engineers from teams in manufacturing industry, academia and telecommunications service operators.

\title{
How to reference
}

In order to correctly reference this scholarly work, feel free to copy and paste the following:

Perrine Berger, Jérôme Bourderionnet, Daniel Dolfi, Fabien Bretenaker and Mehdi Alouini (2011). Slow and Fast Light in Semiconductor Optical Amplifiers for Microwave Photonics Applications, Advances in Optical Amplifiers, Prof. Paul Urquhart (Ed.), ISBN: 978-953-307-186-2, InTech, Available from:

http://www.intechopen.com/books/advances-in-optical-amplifiers/slow-and-fast-light-in-semiconductor-opticalamplifiers-for-microwave-photonics-applications

\section{INTECH}

open science / open minds

\section{InTech Europe}

University Campus STeP Ri

Slavka Krautzeka 83/A

51000 Rijeka, Croatia

Phone: +385 (51) 770447

Fax: +385 (51) 686166

www.intechopen.com

\section{InTech China}

Unit 405, Office Block, Hotel Equatorial Shanghai

No.65, Yan An Road (West), Shanghai, 200040, China

中国上海市延安西路 65 号上海国际贵都大饭店办公楼 405 单元

Phone: +86-21-62489820

Fax: $+86-21-62489821$ 
(C) 2011 The Author(s). Licensee IntechOpen. This chapter is distributed under the terms of the Creative Commons Attribution-NonCommercialShareAlike-3.0 License, which permits use, distribution and reproduction for non-commercial purposes, provided the original is properly cited and derivative works building on this content are distributed under the same license. 